\title{
Study of the morphology of the region surrounding eHWC J1850+001
}

\author{
Chad Brisbois ${ }^{a, *}$ on behalf of the HAWC Collaboration \\ (a complete list of authors can be found at the end of the proceedings) \\ ${ }^{a}$ University of Maryland College Park, \\ College Park, MD, USA \\ E-mail: chadb@umd.edu
}

\begin{abstract}
Although at extreme energies $(>50 \mathrm{TeV}) \gamma$-ray sources generally have large angular separations from one another as observed on Earth, at lower energies in the galactic plane this is often not the case. HAWC observes extended emission from the source eHWC J1850+001 exceeding 50 $\mathrm{TeV}$, and at lower energies this region appears to consist of multiple sources of $\gamma$-ray emission. These include the 3HWC J1849+001 source but also two nearby H.E.S.S. sources observed in their Galactic Plane Survey. Therefore, a full description of the region requires a morphological study including the full energy range of HAWC data. Understanding the spatial features of the emission in this region is important to associate the sources observations at other wavelengths, which may point to hadronic or leptonic origins for the $\gamma$-ray emission. There are multiple pulsar wind nebulae and super nova remnant systems in the vicinity that may be responsible for the emission in this region, including the pulsar PSR J1849+001 and its pulsar wind nebula, which is a likely candidate for the $>50 \mathrm{TeV}$ energy emission seen by HAWC.
\end{abstract}

$37^{\text {th }}$ International Cosmic Ray Conference (ICRC 2021)

July 12 th - 23rd, 2021

Online - Berlin, Germany

\footnotetext{
${ }^{*}$ Presenter
} 


\section{Introduction}

The VHE source eHWC J1850+001 was identified to emit significant flux above $50 \mathrm{TeV}$ and was fit with a Gaussian extent of $0.37^{\circ} \pm 0.16^{\circ}$ [1]. However, in the region surrounding eHWC J1850+001, significant emission has been observed at lower energies in the High Energy Stereoscopic System (H.E.S.S.) Galactic Plane Survey (hereafter HGPS) and previous catalogs ${ }^{1}$ from the High Altitude Water Cherenkov (HAWC) Collaboration [2-4]. Unlike the HGPS, the 2HWC and 3HWC did not provide a detailed description of the morphology and spectrum of the region, instead using a more simplistic search for isolated sources, both point-like and extended $[2,3]$. The 3 HWC search found four sources in the region, with the HGPS reporting three. With the context of this high energy detection, and multiple sources in the region at lower energies in those catalogs, a detailed investigation of the region testing the likelihood of different models is needed to better interpret the HAWC data.

The H.ES.S. Collaboration in the HGPS identified several (potential) counterparts to the 3 sources it observed in the region [4].

- HESS J1849-000

- Gaussian morphology: $0.09^{\circ} \pm 0.015^{\circ}$ extent

- Associated with PSR J1849-0001 and PWN G32.6+0.5

- HESS J1852-000

- Gaussian morphology: $0.28^{\circ} \pm 0.042^{\circ}$ extent

- Potential Associations:

* SNR G32.8-0.1

* SNR G33.2-0.6

* PSR J1853+0011

* PSR J1853-0004

- HESS J1848-018

- Gaussian morphology: $0.25^{\circ} \pm 0.032^{\circ}$ extent

- Possible Associations:

* Starburst region $\mathrm{W} 43$

* 3FGL J1848.4-0141

In the case of HESS J1852-000, the extent reported in the HGPS is larger than the spatial distribution of the potential sources, and only an instrument with superior angular resolution to HESS and HAWC is likely to enable a firm association to be made at $\mathrm{TeV}$ energies. However, for HESS J1848-018 the possible association with W43 might be confirmed with a hard spectrum extending to $100 \mathrm{TeV}$, indicating the presence of a hadronic accelerator.

${ }^{1}$ The 2 HWC and 3HWC catalogs [2, 3] 


\section{Data \& Methods}

The data used in this study comprises 1343 days of HAWC data, representing 305 days more exposure than the analysis presented in [1]. This analysis is performed using all reconstructed data above $1 \mathrm{TeV}$. The model for the region is developed iteratively. First an empirical model for the diffuse emission is defined assuming a Gaussian function of galactic latitude with a fixed width of $1^{\circ}$, and a power law spectrum with an index fixed at -2.75 . The normalization is left free. Next, an extended source is added with a Gaussian morphology and power law spectrum with all parameters free. This process then repeated until no additional sources were found. In the case of the source near the location of the prior eHWC J1850+001 source, the addition of other extended sources caused it to converge to a small extent. In this paper, the source (hereafter referred to as HAWC J1849) is presented as a point source.

\section{Results}

The model presented here consists of 3 sources (HAWC J1849 and two extended sources) and an empirical fit for the galactic diffuse emission. The spectrum for HAWC J1849 matches well with the spectrum for HESS J1849-000 in the HGPS[4] (as can be seen in Figure 1). From the model presented here, incorporating multiple extended sources, it seems likely that the eHWC J1850+001 extent of $0.37^{\circ} \pm 0.16^{\circ}$ was due to source confusion and it is more likely well-fit as a point source $[1]$.

\section{Conclusions}

The good agreement thus far in the spectrum between HESS J1849-000 and HAWC J1849 is encouraging, and points toward further refinement of the model converging to a much clearer description of the region than previously presented in any HAWC analysis. Additionally, a follow-up study using Fermi-LAT data will explore the possibility of other possible associations near W43 for the TeV emission. Further steps to be taken using HAWC data is running the analysis on an update of the HAWC reconstruction with improved core reconstruction, $\gamma /$ hadron separation, and low energy response. This will allow for a better look into this complex region of the galaxy.

\section{Acknowledgments}

We acknowledge the support from: the US National Science Foundation (NSF); the US Department of Energy Office of High-Energy Physics; the Laboratory Directed Research and Development (LDRD) program of Los Alamos National Laboratory; Consejo Nacional de Ciencia y Tecnología (CONACyT), México, grants 271051, 232656, 260378, 179588, 254964, 258865, 243290, 132197, A1-S-46288, A1-S-22784, cátedras 873, 1563, 341, 323, Red HAWC, México; DGAPA-UNAM grants IG101320, IN111716-3, IN111419, IA102019, IN110621, IN110521; VIEP-BUAP; PIFI 2012, 2013, PROFOCIE 2014, 2015; the University of Wisconsin Alumni Research Foundation; the Institute of Geophysics, Planetary Physics, and Signatures at Los Alamos National Laboratory; Polish Science Centre grant, DEC-2017/27/B/ST9/02272; Coordinación de la Investigación Científica 
de la Universidad Michoacana; Royal Society - Newton Advanced Fellowship 180385; Generalitat Valenciana, grant CIDEGENT/2018/034; Chulalongkorn University's CUniverse (CUAASC) grant; Coordinación General Académica e Innovación (CGAI-UdeG), PRODEP-SEP UDG-CA499; Institute of Cosmic Ray Research (ICRR), University of Tokyo, H.F. acknowledges support by NASA under award number 80GSFC21M0002. We also acknowledge the significant contributions over many years of Stefan Westerhoff, Gaurang Yodh and Arnulfo Zepeda Dominguez, all deceased members of the HAWC collaboration. Thanks to Scott Delay, Luciano Díaz and Eduardo Murrieta for technical support.

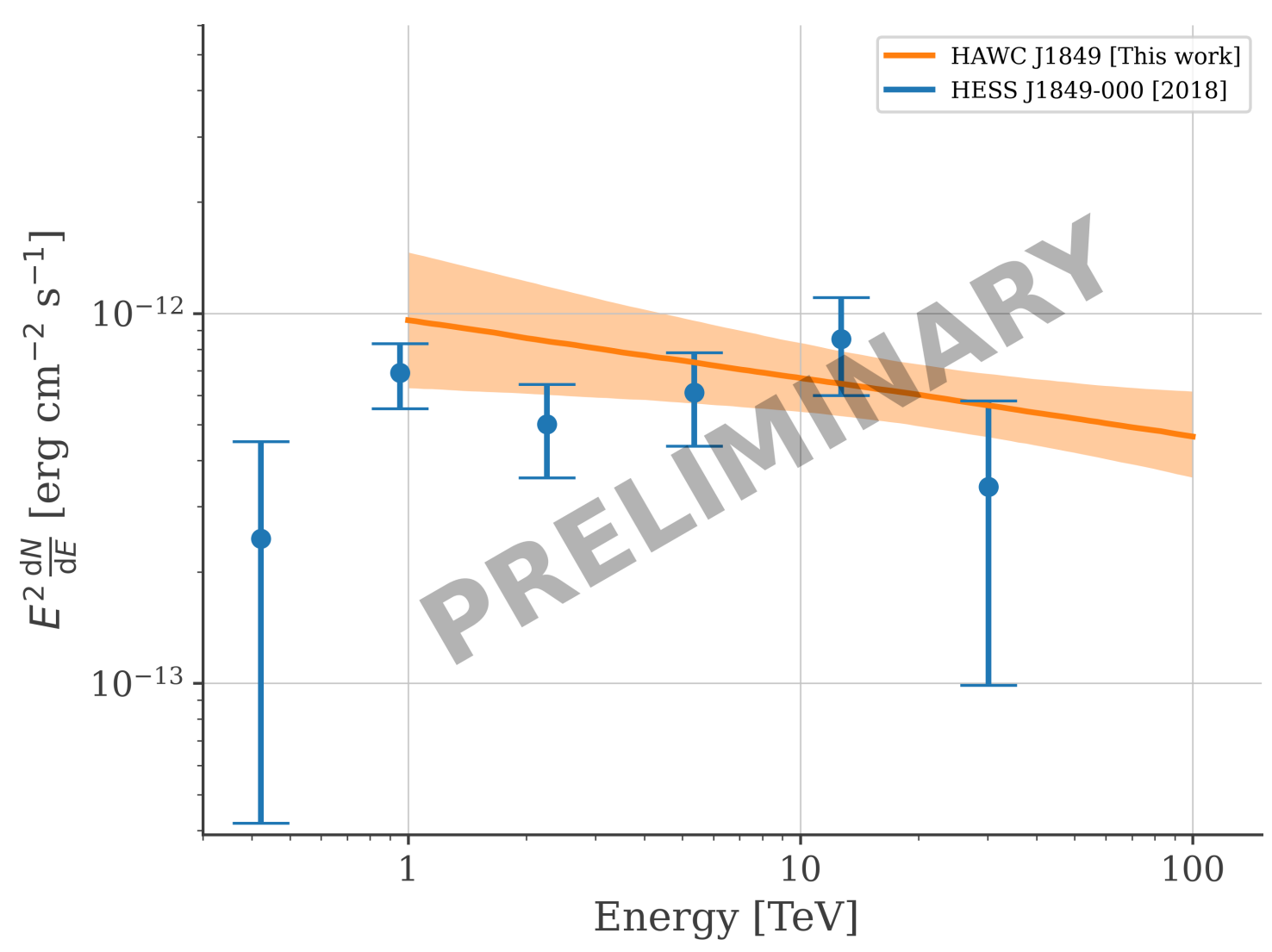

Figure 1: Spectrum of HAWC J1849 fit with a point source morphology. Spectral points from HESS J1849-000 taken from [4].

\section{References}

[1] A.U. Abeysekara, A. Albert, R. Alfaro, J.R.A. Camacho, J.C. Arteaga-Velázquez, K.P. Arunbabu et al., Multiple Galactic Sources with Emission Above $56 \mathrm{TeV}$ Detected by HAWC, Physical Review Letters 124 (2020) .

[2] A.U. Abeysekara, A. Albert, R. Alfaro, C. Alvarez, J.D. Álvarez, R. Arceo et al., The 2HWC HAWC Observatory Gamma-Ray Catalog, The Astrophysical Journal 843 (2017) 40. 
[3] A. Albert, R. Alfaro, C. Alvarez, J.R. Angeles Camacho, J.C. Arteaga-Velzquez, K.P. Arunbabu et al., 3HWC: The Third HAWC Catalog of Very-High-Energy Gamma-ray Sources, jul, 2020. 10.3847/1538-4357/abc2d8.

[4] H. Abdalla, A. Abramowski, F. Aharonian, F. Ait Benkhali, E.O. Angüner, M. Arakawa et al., The H.E.S.S. Galactic plane survey, Astronomy and Astrophysics 612 (2018) A1 [1804.02432].

[5] E.V. Gotthelf, J.P. Halpern, R. Terrier and F. Mattana, Discovery of an energetic $38.5 \mathrm{~ms}$ pulsar powering the Gamma-ray source IGR J18490-0000/HESS J1849-000, Astrophysical Journal Letters 729 (2011) 16 [1012 .2121].

[6] S. Bogdanov, W.C.G. Ho, T. Enoto, S. Guillot, A.K. Harding, G.K. Jaisawal et al., Neutron Star Interior Composition Explorer X-Ray Timing of the Radio and $\gamma$-Ray Quiet Pulsars PSR J1412+7922 and PSR J1849-0001, The Astrophysical Journal 877 (2019) 69 [1902 . 00144]. 


\section{Full Authors List: HAWC Collaboration}

A.U. Abeysekara ${ }^{48}$, A. Albert ${ }^{21}$, R. Alfaro ${ }^{14}$, C. Alvarez $^{41}$, J.D. Álvarez ${ }^{40}$, J.R. Angeles Camacho ${ }^{14}$, J.C. Arteaga-Velázquez ${ }^{40}$, K. P. Arunbabu $^{17}$, D. Avila Rojas ${ }^{14}$, H.A. Ayala Solares ${ }^{28}$, R. Babu ${ }^{25}$, V. Baghmanyan ${ }^{15}$, A.S. Barber ${ }^{48}$, J. Becerra Gonzalez ${ }^{11}$, E. BelmontMoreno $^{14}$, S.Y. BenZvi ${ }^{29}$, D. Berley ${ }^{39}$, C. Brisbois ${ }^{39}$, K.S. Caballero-Mora ${ }^{41}$, T. Capistrán ${ }^{12}$, A. Carramiñana ${ }^{18}$, S. Casanova ${ }^{15}$, O. Chaparro-Amaro $^{3}$, U. Cotti ${ }^{40}$, J. Cotzomi $^{8}$, S. Coutiño de León ${ }^{18}$, E. De la Fuente ${ }^{46}$, C. de León ${ }^{40}$, L. Diaz-Cruz ${ }^{8}$, R. Diaz Hernandez ${ }^{18}$, J.C. Díaz-Vélez ${ }^{46}$, B.L. Dingus ${ }^{21}$, M. Durocher ${ }^{21}$, M.A. DuVernois ${ }^{45}$, R.W. Ellsworth ${ }^{39}$, K. Engel ${ }^{39}$, C. Espinoza ${ }^{14}$, K.L. Fan ${ }^{39}$, K. Fang $^{45}$, M. Fernández Alonso ${ }^{28}$, B. Fick ${ }^{25}$, H. Fleischhack ${ }^{51,11,52}$, J.L. Flores ${ }^{46}$, N.I. Fraija ${ }^{12}$, D. Garcia ${ }^{4}$, J.A. García-González ${ }^{20}$, J. L. García-Luna ${ }^{46}$, G. García-Torales ${ }^{46}$, F. Garfias ${ }^{12}$, G. Giacinti ${ }^{22}$, H. Goksu ${ }^{22}$, M.M. González ${ }^{12}$, J.A. Goodman ${ }^{39}$, J.P. Harding ${ }^{21}$, S. Hernandez ${ }^{14}$, I. Herzog ${ }^{25}$, J. Hinton ${ }^{22}$, B. Hona ${ }^{48}$, D. Huang ${ }^{25}$, F. Hueyotl-Zahuantitla ${ }^{41}$, C.M. Hui ${ }^{23}$, B. Humensky ${ }^{39}$, P. Hüntemeyer ${ }^{25}$, A. Iriarte ${ }^{12}$, A. Jardin-Blicq ${ }^{22,49,50}$, H. Jhee ${ }^{43}$, V. Joshi ${ }^{7}$, D. Kieda ${ }^{48}$, G J. Kunde ${ }^{21}$, S. Kunwar ${ }^{22}$, A. Lara ${ }^{17}$, J. Lee ${ }^{43}$, W.H. Lee ${ }^{12}$, D. Lennarz ${ }^{9}$, H. León Vargas ${ }^{14}$, J. Linnemann ${ }^{24}$, A.L. Longinotti ${ }^{12}$, R. López-Coto ${ }^{19}$, G. Luis-Raya ${ }^{44}$, J. Lundeen ${ }^{24}$, K. Malone ${ }^{21}$, V. Marandon $^{22}$, O. Martinez ${ }^{8}$, I. Martinez-Castellanos ${ }^{39}$, H. Martínez-Huerta ${ }^{38}$, J. Martínez-Castro ${ }^{3}$, J.A.J. Matthews ${ }^{42}$, J. McEnery ${ }^{11}$, P. Miranda-Romagnoli $^{34}$, J.A. Morales-Soto ${ }^{40}$, E. Moreno ${ }^{8}$, M. Mostafá ${ }^{28}$, A. Nayerhoda ${ }^{15}$, L. Nellen ${ }^{13}$, M. Newbold ${ }^{48}$, M.U. Nisa ${ }^{24}$, R. Noriega-Papaqui ${ }^{34}$, L. Olivera-Nieto ${ }^{22}$, N. Omodei ${ }^{32}$, A. Peisker ${ }^{24}$, Y. Pérez Araujo ${ }^{12}$, E.G. Pérez-Pérez ${ }^{44}$, C.D. Rho ${ }^{43}$, C. Rivière ${ }^{39}$, D. Rosa-Gonzalez ${ }^{18}$, E. Ruiz-Velasco ${ }^{22}$, J. Ryan ${ }^{26}$, H. Salazar ${ }^{8}$, F. Salesa Greus ${ }^{15,53}$, A. Sandoval ${ }^{14}$, M. Schneider ${ }^{39}$, H. Schoorlemmer ${ }^{22}$, J. Serna-Franco ${ }^{14}$, G. Sinnis ${ }^{21}$, A.J. Smith ${ }^{39}$, R.W. Springer ${ }^{48}$, P. Surajbali ${ }^{22}$, I. Taboada ${ }^{9}$, M. Tanner ${ }^{28}$, K. Tollefson ${ }^{24}$, I. Torres ${ }^{18}$, R. Torres-Escobedo $^{30}$, R. Turner ${ }^{25}$, F. Ureña-Mena ${ }^{18}$, L. Villaseñor ${ }^{8}$, X. Wang ${ }^{25}$, I.J. Watson ${ }^{43}$, T. Weisgarber ${ }^{45}$, F. Werner ${ }^{22}$, E. Willox ${ }^{39}$, J. Wood ${ }^{23}$, G.B. Yodh ${ }^{35}$, A. Zepeda ${ }^{4}$, H. Zhou ${ }^{30}$

${ }^{1}$ Barnard College, New York, NY, USA, ${ }^{2}$ Department of Chemistry and Physics, California University of Pennsylvania, California, PA, USA, ${ }^{3}$ Centro de Investigación en Computación, Instituto Politécnico Nacional, Ciudad de México, México, ${ }^{4}$ Physics Department, Centro de Investigación y de Estudios Avanzados del IPN, Ciudad de México, México, ${ }^{5}$ Colorado State University, Physics Dept., Fort Collins, CO, USA, ${ }^{6}$ DCI-UDG, Leon, Gto, México, ${ }^{7}$ Erlangen Centre for Astroparticle Physics, Friedrich Alexander Universität, Erlangen, BY, Germany, ${ }^{8}$ Facultad de Ciencias Físico Matemáticas, Benemérita Universidad Autónoma de Puebla, Puebla, México, ${ }^{9}$ School of Physics and Center for Relativistic Astrophysics, Georgia Institute of Technology, Atlanta, GA, USA, ${ }^{10}$ School of Physics Astronomy and Computational Sciences, George Mason University, Fairfax, VA, USA, ${ }^{11}$ NASA Goddard Space Flight Center, Greenbelt, MD, USA, ${ }^{12}$ Instituto de Astronomía, Universidad Nacional Autónoma de México, Ciudad de México, México, ${ }^{13}$ Instituto de Ciencias Nucleares, Universidad Nacional Autónoma de México, Ciudad de México, México, ${ }^{14}$ Instituto de Física, Universidad Nacional Autónoma de México, Ciudad de México, México, ${ }^{15}$ Institute of Nuclear Physics, Polish Academy of Sciences, Krakow, Poland, ${ }^{16}$ Instituto de Física de São Carlos, Universidade de São Paulo, São Carlos, SP, Brasil, ${ }^{17}$ Instituto de Geofísica, Universidad Nacional Autónoma de México, Ciudad de México, México, ${ }^{18}$ Instituto Nacional de Astrofísica, Óptica y Electrónica, Tonantzintla, Puebla, México, ${ }^{19}$ INFN Padova, Padova, Italy, ${ }^{20}$ Tecnologico de Monterrey, Escuela de Ingeniería y Ciencias, Ave. Eugenio Garza Sada 2501, Monterrey, N.L., 64849, México, ${ }^{21}$ Physics Division, Los Alamos National Laboratory, Los Alamos, NM, USA, ${ }^{22}$ Max-Planck Institute for Nuclear Physics, Heidelberg, Germany, ${ }^{23}$ NASA Marshall Space Flight Center, Astrophysics Office, Huntsville, AL, USA, ${ }^{24}$ Department of Physics and Astronomy, Michigan State University, East Lansing, MI, USA, ${ }^{25}$ Department of Physics, Michigan Technological University, Houghton, MI, USA, ${ }^{26}$ Space Science Center, University of New Hampshire, Durham, NH, USA, ${ }^{27}$ The Ohio State University at Lima, Lima, OH, USA, ${ }^{28}$ Department of Physics, Pennsylvania State University, University Park, PA, USA, ${ }^{29}$ Department of Physics and Astronomy, University of Rochester, Rochester, NY, USA, ${ }^{30}$ Tsung-Dao Lee Institute and School of Physics and Astronomy, Shanghai Jiao Tong University, Shanghai, China, ${ }^{31}$ Sungkyunkwan University, Gyeonggi, Rep. of Korea, ${ }^{32}$ Stanford University, Stanford, CA, USA, ${ }^{33}$ Department of Physics and Astronomy, University of Alabama, Tuscaloosa, AL, USA, ${ }^{34}$ Universidad Autónoma del Estado de Hidalgo, Pachuca, Hgo., México, ${ }^{35}$ Department of Physics and Astronomy, University of California, Irvine, Irvine, CA, USA, ${ }^{36}$ Santa Cruz Institute for Particle Physics, University of California, Santa Cruz, Santa Cruz, CA, USA, ${ }^{37}$ Universidad de Costa Rica, San José, Costa Rica, ${ }^{38}$ Department of Physics and Mathematics, Universidad de Monterrey, San Pedro Garza García, N.L., México, ${ }^{39}$ Department of Physics, University of Maryland, College Park, MD, USA, ${ }^{40}$ Instituto de Física y Matemáticas, Universidad Michoacana de San Nicolás de Hidalgo, Morelia, Michoacán, México, ${ }^{41}$ FCFM-MCTP, Universidad Autónoma de Chiapas, Tuxtla Gutiérrez, Chiapas, México, ${ }^{42}$ Department of Physics and Astronomy, University of New Mexico, Albuquerque, NM, USA, ${ }^{43}$ University of Seoul, Seoul, Rep. of Korea, ${ }^{44}$ Universidad Politécnica de Pachuca, Pachuca, Hgo, México, ${ }^{45}$ Department of Physics, University of Wisconsin-Madison, Madison, WI, USA, ${ }^{46}$ CUCEI, CUCEA, Universidad de Guadalajara, Guadalajara, Jalisco, México, ${ }^{47}$ Universität Würzburg, Institute for Theoretical Physics and Astrophysics, Würzburg, Germany, ${ }^{48}$ Department of Physics and Astronomy, University of Utah, Salt Lake City, UT, USA, ${ }^{49}$ Department of Physics, Faculty of Science, Chulalongkorn University, Pathumwan, Bangkok 10330, Thailand, ${ }^{50}$ National Astronomical Research Institute of Thailand (Public Organization), Don Kaeo, MaeRim, Chiang Mai 50180, Thailand, ${ }^{51}$ Department of Physics, Catholic University of America, Washington, DC, USA, ${ }^{52}$ Center for Research and Exploration in Space Science and Technology, NASA/GSFC, Greenbelt, MD, USA, ${ }^{53}$ Instituto de Física Corpuscular, CSIC, Universitat de València, Paterna, Valencia, Spain 